\title{
Convective damping of buoyancy anomalies and its effect on lapse rates in the tropical lower troposphere
}

\author{
I. Folkins \\ Department of Physics and Atmospheric Science, Dalhousie University, Halifax, Nova Scotia, B3H 3J5, Canada
}

Received: 29 July 2005 - Published in Atmos. Chem. Phys. Discuss.: 23 August 2005

Revised: 17 November 2005 - Accepted: 26 November 2005 - Published: 2 January 2006

\begin{abstract}
In regions of the tropics undergoing active deep convection, the variation of lower tropospheric lapse rates $(2.0 \mathrm{~km}$ to $5.2 \mathrm{~km})$ with height is inconsistent with both reversible moist adiabatic and pseudoadiabatic assumptions. It is argued that this anomalous behavior arises from the tendency for the divergence of a convective buoyancy anomaly to be primarily offset by the collective divergence of other updrafts and downdrafts within one Rossby radius of deformation. Ordinarily, convective mass flux divergences are at least partially offset by an induced radiative mass flux divergence in the background atmosphere. If mass flux divergences from lower tropospheric convection are balanced mainly by those of neighboring updrafts/downdrafts, it would force the vertical clear sky radiative mass flux of the background atmosphere to be weakly dependent on height. This is observed at several radiosonde locations in the Western Tropical Pacific between 2.0 and the $5.2 \mathrm{~km}$ melting level. At tropical locations where SST's exceed $27^{\circ} \mathrm{C}$ over a region whose horizontal extent exceeds the local Rossby radius, this condition on the vertical variation of the background radiative mass flux partially constrains the range of physically allowed mean temperature and moisture profiles in the lower troposphere.
\end{abstract}

\section{Introduction}

It is commonly accepted that the mean temperature profile in the tropics approximately follows a moist adiabat. If one starts at the surface with an air parcel with typical values of temperature and relative humidity, and lifts this air parcel upward along a moist adiabatic trajectory, one generates a temperature profile that approximates the tropical mean. It is not obvious why the tropical atmosphere should have this

Correspondence to: I. Folkins

(ian.folkins@dal.ca) property. Unlike the establishment of dry adiabatic layers, this process cannot depend on direct physical mixing, since deep convective updrafts cover only a small fraction of the tropics. Instead, the process is believed to be mediated by gravity waves. Gravity waves propagate outward from buoyancy anomalies such as those associated with deep convection, and give rise to vertical motions in the background atmosphere that modify the density profile of the background atmosphere in such a way as to diminish the buoyancy of the heat source (Bretherton and Smolarkiewicz, 1989). For example, condensational heating within a positively buoyant warm anomaly is communicated to the larger scale background atmosphere by an induced descent, while negatively buoyant cold anomalies cool the atmosphere by induced ascent. A heat source in which the temperature profile followed a moist adiabat would be expected to drive the background atmosphere toward a stratification which was also approximately moist adiabatic.

There are several difficulties with accepting this explanation of the temperature structure of the tropical troposphere. One difficulty is that lapse rates in the lower troposphere of deep convective tropical regions deviate significantly from those predicted by moist adiabatic ascent (Mapes, 2001; Folkins and Martin, 2005). In addition, many observations have shown that updrafts speeds are much smaller than predicted by undilute parcel ascent. Although updrafts are on average slightly positively buoyant, they exhibit a wide range of both positive and negative buoyancies (Jorgensen and LeMone, 1989; Lucas et al., 1994; Wei et al., 1998). These observations presumably reflect the efficiency of entrainment in modifying updraft buoyancies in the lower tropical troposphere, and are inconsistent with the view that updrafts in the lower troposphere are undilute, and as such, would be in a position to impose a moist adiabatic density profile on the background atmosphere. Finally, the density of the lower troposphere is likely to be affected by gravity waves from both updrafts and downdrafts. In the lower

(C) 2006 Author(s). This work is licensed under a Creative Commons License. 
troposphere, the downdraft mass flux is comparable to the updraft mass flux (May and Rajopadhyaya, 1999). The majority of these downdrafts are probably unsaturated, since those originating from the mid-troposphere would be starting out with low values of equivalent potential temperature, and if saturated, would generate much larger negative buoyancies than observed (e.g. Wei et al., 1998). The gravity waves generated by these unsaturated downdrafts would not be expected to drive the atmosphere toward a moist adiabatic temperature profile. The impact of downdrafts on mean tropical temperatures is usually not considered. It would, however, be desirable to have some explanation for the observed density structure of the lower tropical troposphere which treated the effects of updrafts and downdrafts on the density of the background atmosphere in a more symmetric manner.

In this paper, the observed temperature structure of the tropical lower troposphere is attributed to the tendency of updrafts and downdrafts to produce zero net mass flux divergence, when averaged over an appropriate spatial scale. It will be shown that this is the type of constraint that would be expected in the limit that entrainment and detrainment are extremely efficient at damping buoyancy anomalies. The argument relies on a conceptual separation of a deep convective region of the tropical atmosphere into convective and background domains. The term deep convective refers to a tropical region where there is quasi-steady rainfall, and is usually associated with sea surface temperatures which exceed $27^{\circ} \mathrm{C}$ (Folkins and Braun, 2003). The convective domain of a deep convective region is defined as that portion of the atmosphere whose density is significantly different from the regional mean, does not obey hydrostatic balance, and is therefore a source of gravity waves to the background atmosphere. Buoyancy accelerations within the convective domain give rise to vertical velocities that help drive turbulent exchange with the background atmosphere. Density anomalies in the tropics usually originate from condensational heating or evaporative cooling, and are therefore associated with cloud droplets and/or precipitation. The diabatic heating inside the convective portion of the domain will usually be modified by cloud radiative heating, and vertical eddy heat flux divergences associated with turbulent motions.

Let $\overline{\omega_{c}(p)}$ and $\overline{\omega_{b}(p)}$ refer to the vertical pressure velocities within the convective and background domains, horizontally and temporally averaged over some deep convective region. It will be assumed that the only source of diabatic heating to the background atmosphere is clear sky radiative cooling. The vertical velocity of the background atmosphere $\overline{\omega_{b}(p)}$ can then be directly related to the clear sky radiative cooling rate $Q_{r}$, and static stability $\sigma$ via

$\overline{\omega_{b}(p)}=\frac{Q_{r}}{\sigma}$.

This equation assumes that potential temperature surfaces are flat and fixed in space. The static stability is closely related to the lapse rate, and has various equivalent forms, including (e.g. Holton, 1992)

$\sigma=\frac{\left(\Gamma_{d}-\Gamma\right)}{\rho g}=-\frac{T}{\theta} \frac{\partial \theta}{\partial p}$

where $\Gamma$ is the lapse rate $(=-d T / d z)$, and $\Gamma_{d}$ is the dry adiabatic lapse rate.

Let $\overline{\omega_{t}(p)}$ refers to the total vertical velocity of a deep convective region. Then

$\overline{\omega_{t}(p)}=\overline{\omega_{c}(p)}+\overline{\omega_{b}(p)}$.

The mass flux divergence is just the vertical gradient of the vertical velocity (i.e. $\delta_{t}(p)=-\frac{\partial \omega_{t}}{\partial p}$, etc.). This implies

$\overline{\delta_{t}(p)}=\overline{\delta_{c}(p)}+\overline{\delta_{b}(p)}$.

From this equation, we arrive at a constraint on temperatures in the lower troposphere by (i) using ECMWF horizontal winds to show that $\overline{\delta_{t}(p)} \sim 0$ when averaged over spatial scales larger than $2000 \mathrm{~km}$ in the tropical lower troposphere, and (ii) presenting an argument that updrafts and downdrafts in the lower troposphere interact in such a way as to make the convective divergence $\overline{\delta_{c}(p)} \sim 0$ over a similar spatial scale. If true, constraints (i) and (ii) would imply that $\overline{\delta_{b}(p)} \sim 0$ on spatial scales larger than $2000 \mathrm{~km}$, and force the background vertical radiative mass flux $\overline{\omega_{b}(p)}$ to be independent of altitude in the lower troposphere. This is consistent with observations, and enables one to account for the observed vertical variation of static stability, and lapse rate. In this view, a divergence constraint on the convective mass flux gives rise to a background clear sky radiative mass flux which is independent of altitude. Since there are a variety of joint temperature and relative humidity profiles that would give rise to a clear sky radiative mass flux that was independent of altitude, this would serve as a coupled constraint on the covariation of humidity and temperature profiles in the tropical lower troposphere. Temperature and moisture profiles in the tropical lower troposphere are also constrained by the range of physically allowed entrainment and detrainment rates that would make densities within updrafts and downdrafts similar to those of the background atmosphere.

In the next section, the arguments of the previous paragraph are motivated by a discussion of observed lapse rates in deep convective regions. The arguments are then restated in Sects. 3-5. Much of the rest of the paper revolves around a discussion of why one might anticipate the emergence of an entrainment-dominated (or buoyancy-repressed) regime in the lower troposphere $(2 \mathrm{~km}-5.2 \mathrm{~km})$, but not in the midtroposphere $(5.2 \mathrm{~km}-10 \mathrm{~km})$. Sect. 8 discusses uncertainties in our knowledge of the total divergence.

\section{Lapse rate profiles in deep convective regions}

During convective adjustment, gravity waves propagating outward from buoyancy anomalies give rise to net vertical 


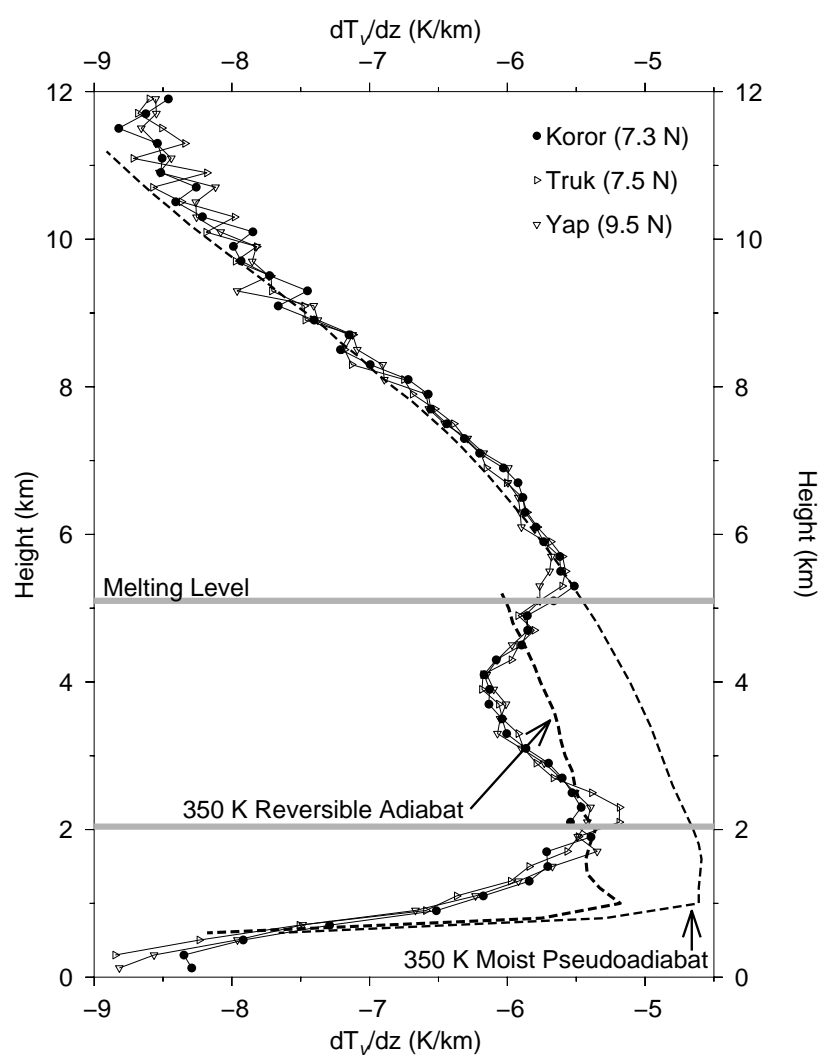

Fig. 1. Annual mean profiles of $d T_{v} / d z$ at 3 radiosonde locations within the Western Tropical Pacific Warm Pool. The profiles were generated from radiosonde measurements, taken up to twice daily from 1999-2001. The thin dashed line is the $d T_{v} / d z$ of a pseudoadiabat starting from the surface with a relative humidity of $80 \%$ and $\theta_{e p}=350 \mathrm{~K}$. The thick dashed line is the $d T_{\rho} / d z$ of a reversible moist adiabat starting from the surface with a relative humidity of $80 \%$ and $\theta_{e}=350 \mathrm{~K}$. The radiosonde data was downloaded from the SPARC (Stratospheric Processes and their Role in Climate) Data Center (http://www.sparc.sunysb.edu).

displacements throughout the background atmosphere which drive the density profile of the atmosphere toward the mean updraft/downdraft density. In addition to temperature and pressure, the density of an air parcel depends on the humidity and condensate loading of the air parcel. The appropriate density variable in the background atmosphere is virtual temperature $T_{v}$. During pseudoadiabatic ascent, where all condensate is assumed to be immediately removed, $T_{v}$ can also be used a density variable. During reversible moist adiabatic ascent, where it is assumed that all condensate is retained by the air parcel, the appropriate density variable is the density temperature $T_{\rho}$. The thin dashed line in Fig. 1 shows the $d T_{v} / d z$ of a moist pseudoadiabat with a pseudoequivalent potential temperature $\left(\theta_{e p}\right)$ (Bolton, 1980) equal to $350 \mathrm{~K}$. This value of $\theta_{e p}$ generates a lapse rate which is in good agreement with observed lapse rates between $5.2 \mathrm{~km}$ and $10 \mathrm{~km}$. The thick dashed line in Fig. 1 shows the $d T_{\rho} / d z$

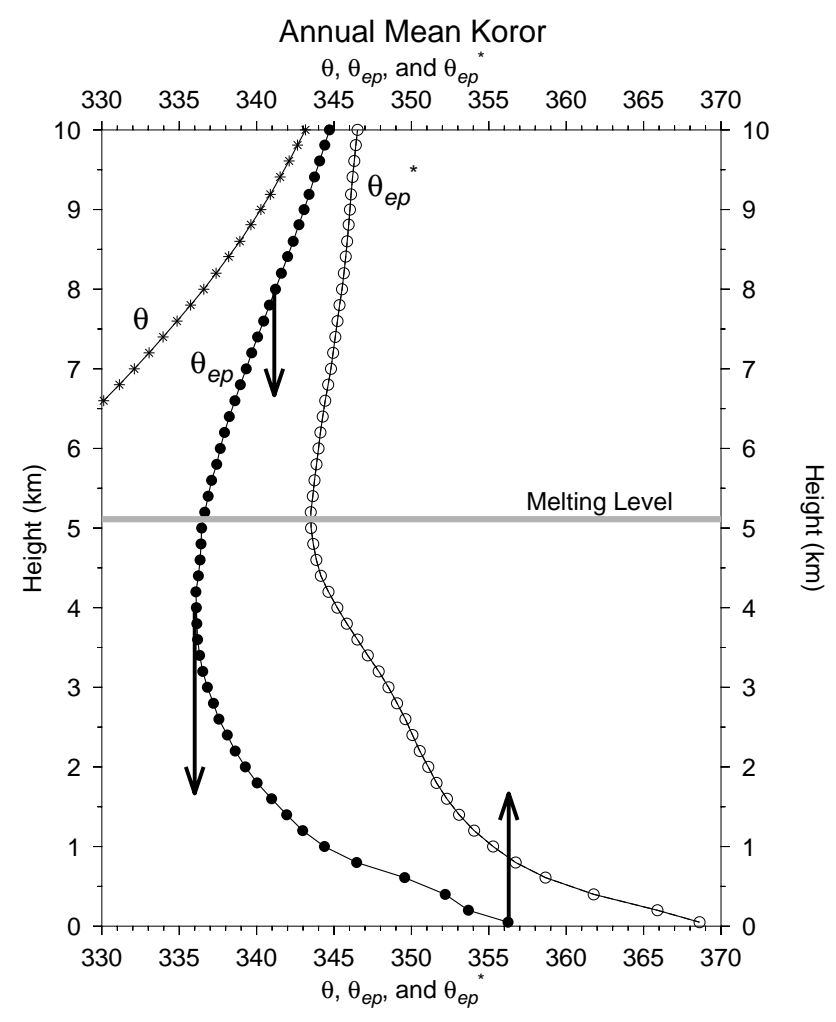

Fig. 2. The annual mean potential temperature ( $\theta$, asterisks), pseudoequivalent potential temperature $\left(\theta_{e p}\right.$, solid circles), and saturated pseudoequivalent potential temperature $\left(\theta_{e p}^{*}\right.$, squares $)$ at Koror. Note that the melting level occurs near the minimum in $\theta_{e p}^{*}$.

of reversible moist adiabat with $\theta_{e}=350 \mathrm{~K}$. (The reversible density temperature was not calculated above the melting level because doing so would require an assumption about the phase of the condensate. In any event, the assumption that all condensate is retained by the air parcel can be expected to become increasingly unrealistic in the upper troposphere.) Below the melting level, the reversible adiabat is in better agreement with the observed lapse rate than the pseudoadiabat (see also Betts, 1982; Xu and Emanuel, 1989). However, neither the reversible or pseudoadiabatic idealizations are able to reproduce the shape of the observed notch in $d T_{v} / d z$ between $2 \mathrm{~km}$ and $5.2 \mathrm{~km}$ (Folkins and Martin, 2005).

Convective adjustment involves an interaction between a buoyancy anomaly and the background atmosphere. One can think of an updraft or downdraft as forcing the background atmosphere to adopt some preferred density profile (e.g. moist adiabatic). This perspective is one-sided, however, because the adjustment process necessarily involves the turbulent exchange of air between an updraft/downdraft and the background atmosphere, during which, the density of the updraft/downdraft can be significantly altered. It has been shown, using both analytic approaches and two- 


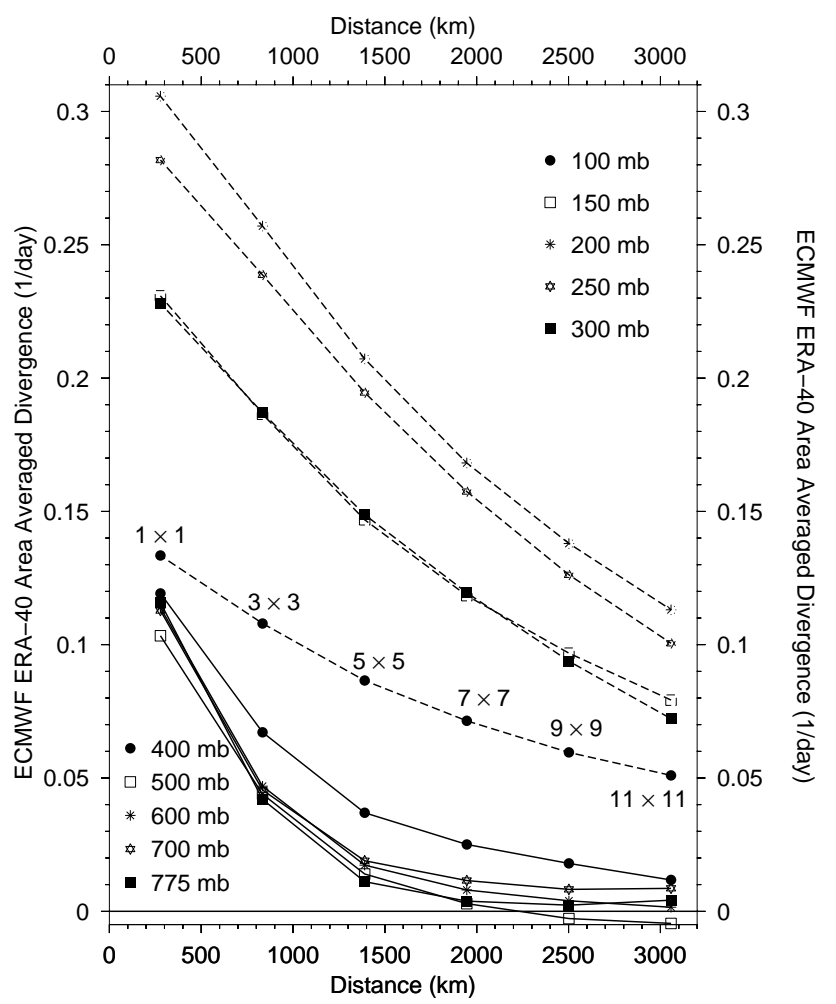

Fig. 3. The rate at which the total divergence from the April 2000 ECMWF ERA-40 analysis decreases as it is spatially averaged over a larger distance. The divergence was calculated by starting at grid cells between $10^{\circ} \mathrm{S}$ and $10^{\circ} \mathrm{N}$ whose divergence was positive, and averaging over progressively larger boxes, centered at the original grid cell, of $3 \times 3,5 \times 5,7 \times 7,9 \times 9$, and $11 \times 11$ grid cells. The spatial scale of a divergence anomaly increases rapidly in going from $400 \mathrm{mb}$ to $300 \mathrm{mb}$. The dashed curves refer to divergences from the $300 \mathrm{mb}$ surface and above.

dimensional cloud modeling, and it is now widely accepted, that updrafts typically entrain air from their environment at altitudes where their buoyancy is increasing, and detrain air into their environment at altitudes where their buoyancy is diminishing (Bretherton and Smolarkiewicz, 1989). The height ranges of preferred entrainment or detrainment can be identified from profiles of $\theta_{e p}$ and saturated pseudoequivalent potential temperature $\left(\theta_{e p}^{*}\right)$. Figure 2 shows annual mean profiles of $\theta_{e p}$ and $\theta_{e p}^{*}$ above Koror. Once a rising air parcel has crossed its lifting condensation level and is saturated, its buoyancy is proportional to the difference between its $\theta_{e p}$ and the $\theta_{e p}^{*}$ of the background atmosphere. (This assumes that the effects of water vapor and condensate loading on density are small. In this case, saturated equivalent potential temperature can be used as a density variable since two air parcels on the same pressure level with the same $\theta_{e p}^{*}$ will have the same temperature). The $\theta_{e p}$ of an air parcel is approximately conserved during saturated and unsaturated vertical displacements. As shown in Fig. 2, an air parcel lifted upward from the surface with $\theta_{e p}=356 \mathrm{~K}$ is initially negatively buoyant. However, once an air parcel crosses its Level of Free Convection (LFC) near $0.7 \mathrm{~km}$, its $\theta_{e p}$ diverges from the background $\theta_{e p}^{*}$, and the air parcel becomes progressively more positively buoyant. In the lower troposphere, therefore, most updrafts will entrain air from their environment. Above the melting level, where $\theta_{e p}^{*}$ gradually increases with height, updrafts will slowly detrain air into the background atmosphere as they become progressively less buoyant.

By the same reasoning, downdrafts would be expected to detrain air into the background atmosphere above the melting level, while entraining air from the background atmosphere below the melting level. Figure 2 shows that a downdraft originating at $8 \mathrm{~km}$ will lose negative buoyancy as it approaches the $\theta_{e p}^{*}$ curve, while an undilute downdraft originating near $4 \mathrm{~km}$ will gain negative buoyancy as it diverges from the $\theta_{e p}^{*}$ curve.

It was mentioned earlier that, in the standard view, one thinks of convective adjustment as driving the atmosphere toward some preferred density profile equal to the mean updraft/downdraft density. This is a simplification, however, because convective plumes which maintained a fixed buoyancy offset from the background atmosphere could, in principle, have zero net mass exchange with the background atmosphere. In this case, one would think of updrafts/downdrafts as imposing a vertical density gradient (or virtual temperature lapse rate) on the background atmosphere, rather than an absolute density profile. For example, Fig. 1 shows that the $\theta_{e p}$ which best describes the lapse rate between $5.2 \mathrm{~km}$ and $10 \mathrm{~km}$ is $350 \mathrm{~K}$, a value which presumably reflects the mass weighted $\theta_{e p}$ of updrafts in this interval. However, Fig. 2 shows that the mean $\theta_{e p}^{*}$ of the background atmosphere increases from roughly $344 \mathrm{~K}$ to $346 \mathrm{~K}$ in this height range. There is therefore a 4-6 K offset between the $\theta_{e p}$ of the pseudoadiabat from the surface which best approximates temperatures between $5.2 \mathrm{~km}$ and $10 \mathrm{~km}$ and the $\theta_{e p}$ which best approximates the lapse rate. The magnitude of this offset may reflect the mean positive buoyancies of updrafts in this region of the troposphere.

\section{The total divergence}

Divergence patterns have a larger horizontal scale in the upper troposphere than in the lower troposphere. Figure 3 shows the rate at which the area averaged divergence decreases if, starting from an initial grid cell in which the divergence is positive, one averages progressively outward over larger and larger squares centered on the original grid cell. Only starting grid cells between $10^{\circ} \mathrm{S}$ and $10^{\circ} \mathrm{N}$ were considered. Divergence values were taken from the ERA40 monthly daily mean ECMWF reanalysis for April 2000 (other months are similar). The ERA-40 reanalysis has a $2.5^{\circ}$ zonal resolution, and a $2.25^{\circ}$ meridional resolution. Divergences in the upper troposphere are stronger, and have 
a much larger spatial extent, than in the lower troposphere. Between $300 \mathrm{mb}$ and $100 \mathrm{mb}$, the spatial scale over which a positive divergence anomaly declines by $1 / \mathrm{e}$ is typically $3000 \mathrm{~km}$. Below $500 \mathrm{mb}$, this distance is typically $900 \mathrm{~km}$. That is, a $3 \times 3$ box of grid cells, centered on a grid cell with positive divergence, typically has $1 / \mathrm{e}$ of the area averaged divergence of the center grid cell. Though not shown in Fig. 3, the distance over which a divergence anomaly declines by $1 / \mathrm{e}$, when starting out with a negative value of divergence, is also roughly equal to $900 \mathrm{~km}$.

The gravity waves propagating outward from a convective source give rise to vertical displacements which perturb the temperature of the background atmosphere. The horizontal distance to which these temperature perturbations extend is referred to as the Rossby radius of deformation. One would expect the spatial scale of a divergence pattern arising from an updraft/downdraft to be approximately equal to the Rossby radius of the gravity waves which have the most influence on background vertical velocities (temperatures). The Rossby radius of a gravity wave is approximately given by $\lambda_{r}=c / f$, where $c$ is the gravity wave speed and $f$ is the Coriolis parameter. Convective sources generate gravity waves with a variety of speeds, so that such sources cannot be characterized by a single Rossby radius. It has been suggested, however, that deep convective sources preferentially generate gravity waves with speeds of $c \sim 24 \mathrm{~m} / \mathrm{s}$ and $c \sim 50 \mathrm{~m} / \mathrm{s}$ (Mapes and Houze, 1995). At a latitude of $10^{\circ}$, these wave speeds corresponds to Rossby deformation radii of $960 \mathrm{~km}$ and $2000 \mathrm{~km}$. The proximity of $960 \mathrm{~km}$ to the lower tropospheric e-folding distance of $900 \mathrm{~km}$ is consistent with the view that the spatial variability of the divergence field in the lower troposphere is dominated by the slower, shorter vertical wavelength stratiform mode.

The spatial resolution of the ECMWF ERA-40 model $(278 \mathrm{~km}$ at the equator) is comparable with the expected coherence length of lower tropospheric divergences $(\sim 960 \mathrm{~km})$. The rate at which the lower tropospheric area averaged divergence decreases is almost as rapid as if positive and negative divergences had been randomly distributed in space. In the upper troposphere, the coherence length of upper tropospheric divergence patterns is much larger than the grid scale of the model, reflecting a much stronger tendency for positive divergences to be preferentially co-located.

\section{The convective divergence}

Figure 3 shows that, between $775 \mathrm{mb}$ and $500 \mathrm{mb}$, the magnitude of the total ECMWF ERA-40 total divergence has a magnitude $\overline{\delta_{t}(p)} \sim 0.01 \mathrm{day}^{-1}$ when area averaged over spatial scales larger than $2000 \mathrm{~km}$. In deep convective regions of the tropics, most of the total divergence originates from vertical motions associated with convective heating and cooling. In this section, it is argued that the weakness of the spatially averaged total divergence in the lower troposphere is due to

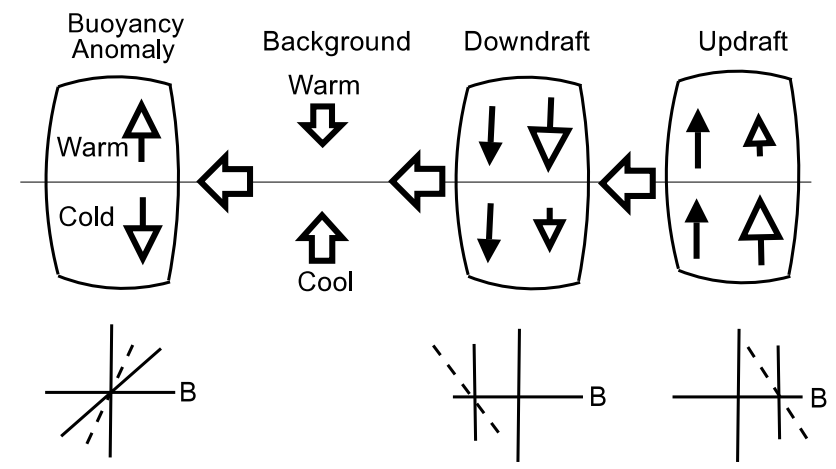

Fig. 4. A schematic diagram showing how an idealized buoyancy anomaly (warm/cold dipole) interacts with the background atmosphere and with surrounding updrafts and downdrafts. There is a nearby downdraft of constant initial negative buoyancy and mass flux, and a nearby updraft of constant initial positive buoyancy and mass flux. Solid arrows indicate mass fluxes prior to the introduction of the buoyancy anomaly, while open arrows indicate mass fluxes after the introduction of the buoyancy anomaly. The size of the arrow gives an approximate indication of the magnitude of the mass flux. The plots along the bottom indicate how buoyancy varies with height inside the buoyancy anomaly and inside the updraft/downdraft. Solid lines refer to buoyancies before the atmosphere responds to the buoyancy anomaly, while dashed lines refer to buoyancies after the atmosphere has responded to the buoyancy anomaly. The initial vertical mass flux within the buoyancy anomaly is zero. The warm upper part of the anomaly induces descent in the background atmosphere, while the cold lower part of the anomaly induces ascent. These vertical motions would lead to a divergent outflow of mass from the background atmosphere toward the buoyancy anomaly. Such a buoyancy anomaly would therefore tend to entrain air from the background atmosphere. Alternatively, buoyancy anomalies whose buoyancy increases with height tend to be convergent. Because the background atmosphere warms (sinks) where the buoyancy anomaly is warm, and cools (ascends) where the anomaly is cold, the magnitude of the buoyancy within the anomaly is reduced via this interaction. The convergent inflow toward the anomaly can also be supplied by an increase in the divergent outflow from surrounding updrafts/downdrafts. The warming of the atmosphere in the upper part of the downdraft would increase its negative buoyancy and downward mass flux (open arrow larger than solid arrow). In the lower part of the downdraft, the cooling of the atmosphere would decrease the downdrafts negative buoyancy and mass flux (open arrow smaller than solid arrow). The downdraft would therefore become divergent. By a similar reasoning the initially nondivergent updraft would also become divergent.

a tendency for the divergences of nearby (within one Rossby radius) updrafts and downdrafts to offset each another.

In a hypothetical atmosphere where the the buoyancy of every updraft/downdraft was constant, there would be no requirement for a net exchange of mass between updrafts/downdrafts and the background atmosphere. Suppose, however, that one introduced into this atmosphere a buoyancy anomaly whose buoyancy increased with height from 


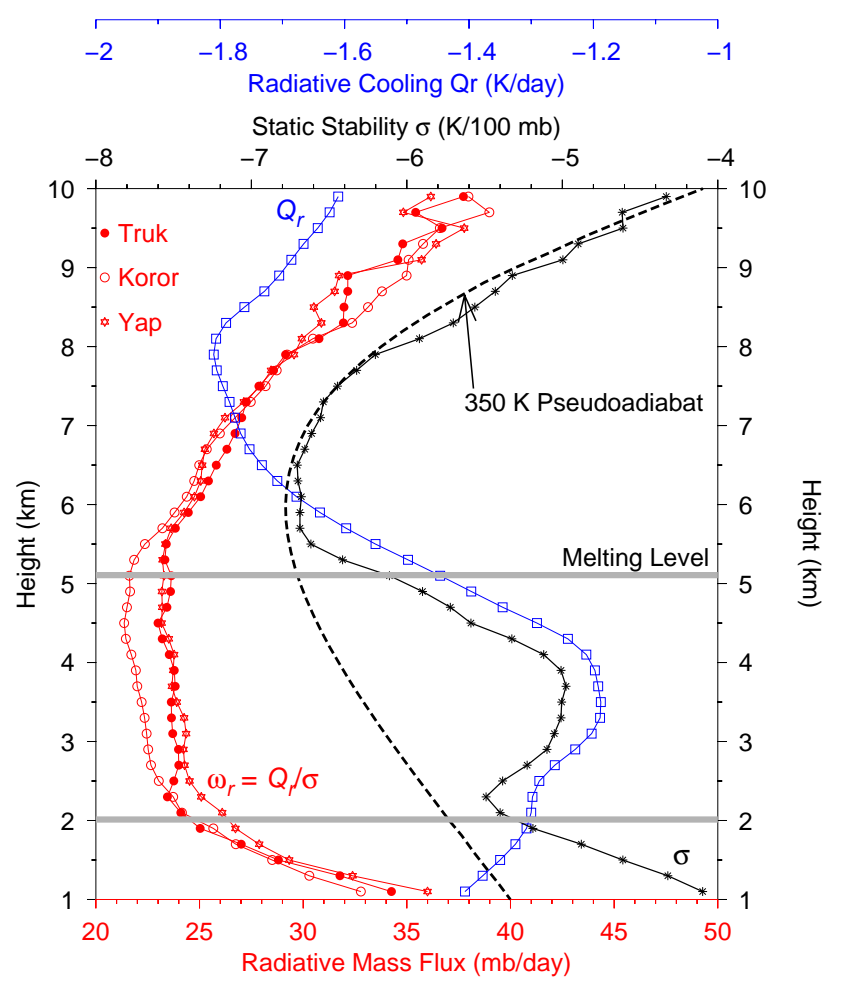

Fig. 5. The black line with asterisks is the annual mean static stability $\sigma$ at Truk. The dashed line is the static stability of a $\theta_{e p}=350 \mathrm{~K}$ moist pseudoadiabat. The blue line is the annual mean clear sky radiative heating rate $Q_{r}$ calculated from seasonal mean temperature and water vapor profiles at Truk, and tropical mean ozone profiles. It was calculated using a four stream, correlated-k distribution, radiative transfer code (Fu and Liou, 1992). The three red lines refer to the clear sky radiative mass flux $\omega_{r}$ at Truk, Koror, and Yap, calculated from the annual mean $\sigma$ and $Q_{r}$ profiles at each station.

negative to positive. The horizontal and vertical redistribution of mass in the background atmosphere associated with such an anomaly is shown in Fig. 4 (Bretherton and Smolarkiewicz, 1989). There would be a downward vertical velocity in the background atmosphere at heights where the buoyancy of the anomaly was positive, and an upward vertical velocity in the background atmosphere at heights where the buoyancy of the anomaly was negative. These induced vertical velocities would be associated with a convergent influx of air toward the anomaly.

The background vertical motions introduced by a heat source change the temperature of the background atmosphere in such a way as to suppress its buoyancy. For example, the downward background motion where the buoyancy of the anomaly is positive warms the atmosphere. Conversely, the upward background motion where the buoyancy of the anomaly is negative cools the atmosphere. These temperature changes reduce the magnitude of the buoyancy within the heat source, reduce its vertical buoyancy gradient, and reduce its induced convergent inflow. This effect could be referred to as self damping.
The temperature changes of the background atmosphere also affect the buoyancies of surrounding updrafts and downdrafts. A warmer background atmosphere would increase the negative buoyancy of the upper half of the downdraft, while a colder background atmosphere would decrease the negative buoyancy of the lower half of the downdraft. A downdraft whose negative buoyancy decreased toward the surface would tend to detrain air, and be associated with a divergent outflow. Similarly, the background temperatures changes would also give rise to buoyancy changes in surrounding updrafts which would contribute toward a divergent outflow of air.

These examples suggest that the mass flux divergence of a heat source should be collectively damped by the divergences of surrounding updrafts and downdrafts. The response shown in Fig. 4 could be characterized as the dry response, since it does not directly consider the effects of cloud microphysics on buoyancy. These effects can, however, be quite significant. In the case of dry convection, the entrainment of colder environmental air into the updraft would diminish the buoyancy (warm bias) of the updraft, but leaves its buoyancy flux (product of the mass flux and the buoyancy) unchanged (Emanuel and Bister, 1996). In the case of moist convection, however, the effect of entrainment on the buoyancy of an updraft is a strong function of the condensate loading of the updraft, the relative humidity of the entrained air, and the temperature. The evaporation of liquid water or ice within the updraft, due to dry air entrainment, can rapidly cool the updraft, and in some cases, change its buoyancy from positive to negative. Buoyancy reversal is more likely in cases of high condensate loading and lower background relative humidity. In general, it is also more likely at warmer temperatures, where for a given background relative humidity and entrainment rate, more evaporation of condensate can occur. This self damping of a moist updraft due to the entrainment of unsaturated air represents an additional negative feedback on the strength of a convergence anomaly.

The effect of entrainment on the buoyancy of a downdraft is more complicated than that of an updraft. In general, it would be expected to reduce the negative buoyancy of a downdraft, since in the lower troposphere, one would usually be introducing air with a higher $\theta_{e}$. However, in the case of a nearly saturated downdraft, the introduction of unsaturated air would increase evaporative cooling, and increase the negative buoyancy of the downdraft. The overall effect of entrainment on the negative buoyancy of a downdraft is probably determined by whether the entrained mixture has a higher or lower relative humidity than the unmixed air parcel. In areas of the tropical ocean where there is frequent deep convection and background relative humidities are quite high, entrainment can be expected to decrease downdraft buoyancies. 


\section{The background divergence}

Induced descent warms the atmosphere and increases radiative cooling rates. Induced ascent cools the atmosphere and decreases radiative cooling rates. In Fig. 4, one would expect the downward motion of the background atmosphere to increase the downward radiative mass flux above the convergence anomaly, and for the upward motion below the convergence anomaly to decrease the downward radiative mass flux. These changes would imply a divergent radiative mass flux in the background atmosphere, which would also partially compensate the mass flux convergence of the buoyancy anomaly.

Climatological profiles of the radiative cooling rates and static stability in deep convective regions suggest that the damping of convective divergence anomalies by an induced radiative mass flux divergence in the lower troposphere is weak. Figure 5 shows the annual mean profile of static stability at Truk. The dashed line indicates the static stability profile generated by undilute pseudoadiabatic ascent from the surface, starting with an air parcel with pseudoequivalent potential temperature $\theta_{e p}$ equal to $350 \mathrm{~K}$. As would be anticipated from Fig. 1, it closely matches the observed static stability above the melting level, but fails to reproduce the notch in the observed $\sigma$ between $2 \mathrm{~km}$ and $5.2 \mathrm{~km}$. The blue curve in Fig. 5 is the rate of clear sky radiative cooling at Truk. It was obtained by averaging over the four seasonal mean $Q_{r}$ profiles at Truk, each calculated from seasonal mean temperature, pressure, and relative humidity profiles. The heating rates calculated by the radiative transfer model (Fu and Liou, 1992) exhibit excellent agreement with line by line calculations (Gettelman et al., 2004). Roughly 500 radiosonde profiles were used to construct each seasonal mean. Below the melting level, the variation with altitude of the static stability and radiative cooling rate is very similar. This gives rise to a radiative mass flux which is roughly constant between $2 \mathrm{~km}$ and $5.2 \mathrm{~km}$. The climatological clear sky radiative mass flux divergence at these locations is therefore small $\left(\delta_{r}(p) \sim 0\right)$. It would also imply that the area averaged divergence of the background atmosphere is small $\left(\overline{\delta_{b}(p)} \sim 0\right)$, provided the $\omega_{r}(p)$ profiles shown in Fig. 5 are representative of other deep convective locations, and the only source of diabatic heating in the background atmosphere is clear sky radiative heating.

In principle, the presence of clouds could significantly perturb lower tropospheric radiative heating rates from their clear sky values. It is likely, however, that effects of cloud radiative heating are concentrated within the clouds themselves. As such, cloud radiative heating would affect cloud buoyancies, but have a reduced impact on the diabatic heating of the background atmosphere. It can be shown, for example, that the vertical gradients of the clear sky heating rates shown in Fig. 5 are only weakly sensitive to the addition of an overhead cirrus anvil. It has also been noted that the clear sky component of radiative heating rates are essentially insen- sitive to different cloud distribution assumptions (Bergman and Hendon, 1998). However, full resolution of this issue is dependent on progress using three-dimensional radiative transfer models with realistic cloud fields (e.g. Giuseppe and Tompkins, 2003).

\section{Origin of lower tropospheric minimum in radiative cooling}

Radiative heating rates in the lower tropical troposphere are quite sensitive to specific humidity, but also to temperature, so that the vertical variation of $Q_{r}$ depends, in part, on the vertical variation of $\sigma$. This leads to some ambiguity in how to interpret a radiative mass flux $\omega_{r}(p)$ that is independent of altitude. For example, the lower tropospheric radiative cooling minimum shown in Fig. 5 could, in principle, be forced by the minimum in static stability. In this case, one might expect $\omega_{r}(p)=Q_{r} / \sigma$ to be independent of height, and the constancy of $\omega_{r}(p)$ between $2 \mathrm{~km}$ and $5.2 \mathrm{~km}$ to be an anticipated response to a particular temperature profile. This would be more likely if $Q_{r}$ and $\sigma$ were tightly coupled. On the other hand, if $Q_{r}$ and $\sigma$ were essentially independent of each other, it seems unlikely that they would covary with height in such a similar way, and much more likely that temperature and specific humidity were mutually adjusting to satisfy an independent constraint on the radiative mass flux. In this section, it is shown that the radiative cooling minimum in the lower tropical troposphere is largely decoupled from the minimum in static stability.

To test whether the lower tropospheric minimum in radiative cooling was forced by the static stability minimum, we constructed a test temperature profile which was as close as possible to a real tropical temperature profile (the mean September-November profile at Koror), but in which the notch in static stability was removed. The dashed line in Fig. 6 shows the difference $T_{t}-T_{\text {Koror }}$ between the test and mean Koror temperature profiles. Below $1.8 \mathrm{~km}$, and above $7.2 \mathrm{~km}$, the test temperature profile was set equal to the Koror profile. Between $1.8 \mathrm{~km}$ and $7.2 \mathrm{~km}$, the lapse rate of the test profile was forced to vary linearly with height. The temperature difference between the two profiles is everywhere less than $0.4 \mathrm{~K}$. The radiative cooling rate of the "smoother" test profile is compared with that of the original Koror profile in Fig. 7. The differences are quite small, with the lower tropospheric cooling minimum slightly deeper for the test case. This demonstrates that the lower tropospheric minimum in the cooling rate does not originate from the minimum in the static stability.

The test described in the previous paragraph confirms expectations from previous work that the radiative cooling minimum in the lower tropical troposphere is largely due to the low transmissivity to space of water vapor in this region of the atmosphere. Longwave radiative cooling rates in the lower troposphere are well reproduced by the "cooling to 


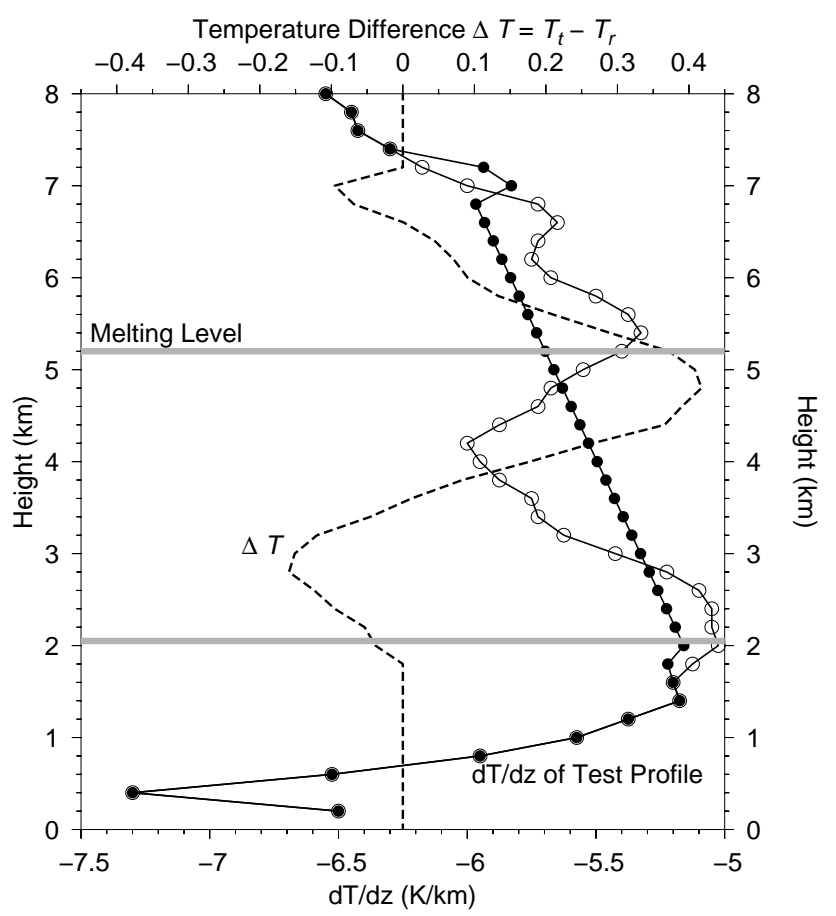

Fig. 6. The curve with open circles is the $d T / d z$ of a reference September-November temperature profile at Koror. The curve with solid circles is the $d T / d z$ of a test temperature profile in which the temperature is kept unchanged below $1.8 \mathrm{~km}$ and above $7.2 \mathrm{~km}$, but interpolated between these two heights in such a way as to give rise to a lapse rate that varies linearly with altitude. The dashed line shows the difference between the test $\left(T_{t}\right)$ and reference $\left(T_{r}\right)$ temperature profiles.

space" approximation (Rodgers and Walshaw, 1966), which can be written (Mapes and Zuidema, 1996)

$Q_{r}(z)=B(z) \sigma(z) T(z, \infty)$,

where $B(z)$ is the Planck function, $\sigma(z)$ is the extinction cross-section (proportional to the humidity), and $T(z, \infty)$ is the transmission between height $z$ and space. The higher temperatures and increased humidities of the lower tropical troposphere would tend to increase radiative cooling rates. However, this is more than offset by a rapid decrease in the transmissivity to space below $8 \mathrm{~km}$ (due to the increase in the overhead column of water vapor), leading to a reduced cooling rates in the lower troposphere (Doherty and Newell, 1984).

\section{Timescales of radiative and convective damping}

It has been argued that, in the lower tropical troposphere, the damping of mass flux divergences from buoyancy anomalies by surrounding convective elements, and the requirement that the total divergence go to zero on spatial scales comparable with the Rossby radius of deformation, force the mean

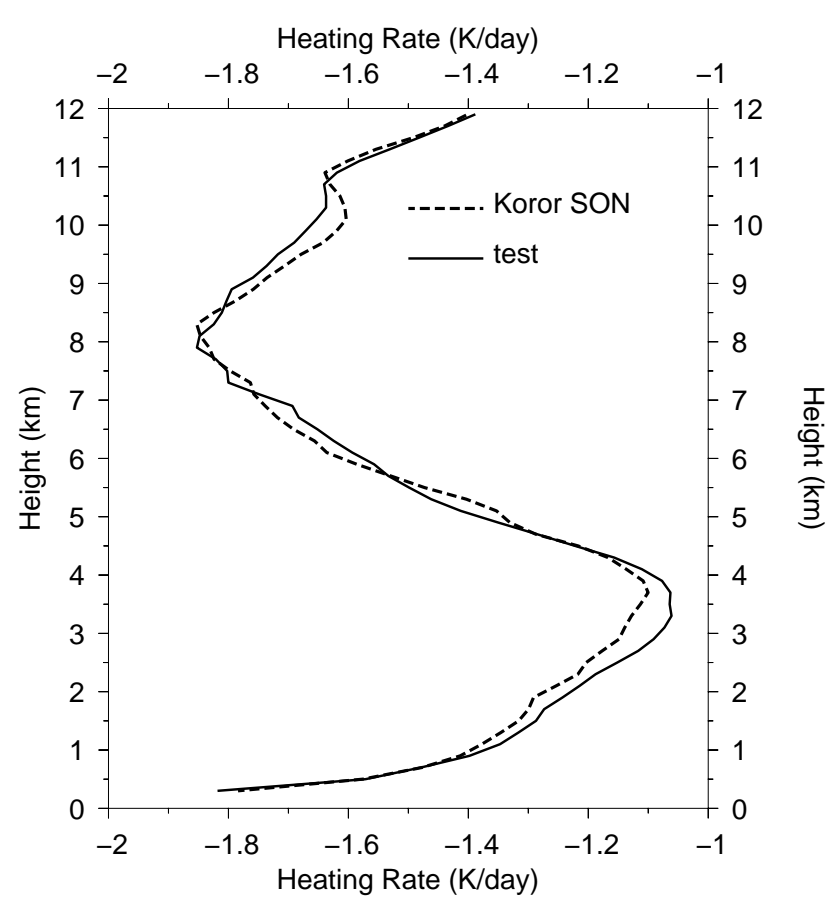

Fig. 7. The solid curve shows the clear sky radiative heating rate of the mean September-November temperature profile at Koror. The dashed curve is the radiative heating rate for the temperature profile, adjusted as indicated in Fig. 6.

clear sky radiative mass flux to be independent of altitude. This would force temperatures and humidities in the lower troposphere to mutually adjust to satisfy a constraint on the radiative mass flux. The degree to which buoyancy anomalies (and their associated divergences) are damped by convective versus radiative processes will depend on the respective timescales of each process. The observed weakness of buoyancy anomalies in the lower tropical troposphere (Jorgensen and LeMone, 1989; Lucas et al., 1994; Wei et al., 1998) is consistent with the view that the timescale for the total damping by both processes is very short. However, no argument has been advanced here as to why the timescale for the divergence of a buoyancy anomaly to be convectively damped should be faster than the timescale for its divergence to be radiatively damped. There has also been no discussion as to why an apparent transition between the convective and radiatively damped regimes occurs near the melting level. Although definitive answers to these questions are beyond the scope of this paper, one can identify some factors which might contribute to their resolution.

The convective damping timescale will depend on the timescale over which the mass fluxes inside updrafts and downdrafts respond to the vertical motions and temperature fluctuations in the background atmosphere from a preexisting updraft/downdraft. The melting level occurs near the minimum in $\theta_{e}^{*}$, which denotes a transition from a conditionally unstable to stable atmosphere. In a conditionally 
unstable atmosphere, a saturated air parcel will immediately become positively buoyant when subjected to an upward positive velocity. It has positive Convective Available Potential Energy (CAPE) and zero Convective Inhibition (CIN). Although subsaturated air parcels in a conditionally unstable atmosphere will have a non-zero convective inhibition, temperature fluctuations can still trigger positive buoyancies in air parcels if the relative humidity of the air parcel is sufficiently high. Near the equator, where the Rossby radius is quite large, temperature perturbations from a buoyancy anomaly extend over a broad area. The likelihood that such a perturbation will trigger an updraft is therefore quite high, although clearly, it would be very sensitive to the background relative humidity.

The situation above the melting level is quite different. In general, it is not possible for an air parcel in an atmosphere where $\theta_{e p}^{*}$ increases monotonically with height to have positive CAPE. There is an intrinsic upper limit on the upward cloud mass flux, set by the number of pre-existing air parcels at that height that are already positively buoyant.

One would would also expect the downdraft mass flux to be more responsive to temperature fluctuations in a conditionally unstable atmosphere. Figure 2 shows that the $\theta_{e p}$ trajectory of an undilute downdraft below the melting level diverges from the $\theta_{e p}^{*}$ curve. From a thermodynamic perspective, every air parcel below the $\theta_{e p}^{*}$ minimum is capable of descending to the surface given sufficient exposure to precipitation. Because the relative humidity of an undilute downdraft decreases as it descends, its ability to evaporate condensate is maintained. In contrast, the $\theta_{e}$ trajectory of an undilute downdraft above the melting level tends to approach the $\theta_{e p}^{*}$ curve. At this point, it becomes saturated and unable to undergo further evaporative cooling. The existence of downdrafts above the melting level therefore depends on a continual resupply of dry air to convective regions by the large scale circulation (exception to this argument will occur in situations of high condensate loading). The transition from a conditionally stable to unstable atmosphere at the melting level may account for the rapid increase in downdraft mass flux near this height (May and Rajopadhyaya, 1999).

Updrafts whose buoyancy is increasing, and which contain condensate, are also subject to self damping due to dry air entrainment. In the atmosphere, the saturated water vapor pressure decreases with height much more rapidly than the density. This means that water vapor phase changes have the potential to have a much greater relative impact on the heat budget of an air parcel in the lower troposphere. One would therefore expect the evaporation or condensation of condensate associated with dry air entrainment to have a bigger impact on reducing the magnitude of buoyancy anomalies in the lower troposphere.
Annual mean GPCP Rainfall (mm/day) 1991 - 2000
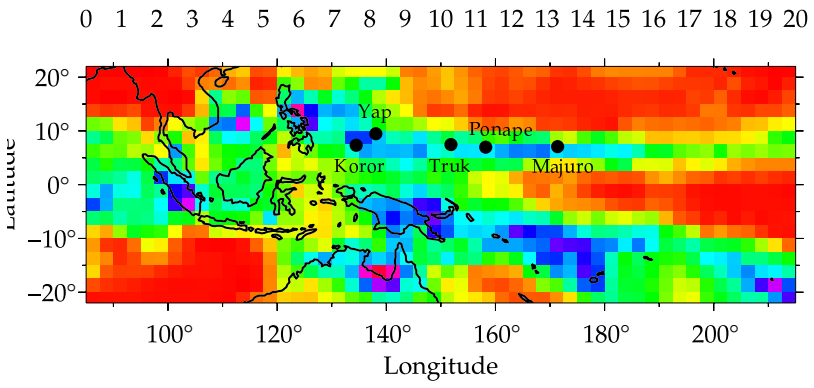

Fig. 8. The locations of the five high resolution radiosonde stations discussed in this paper, superimposed on a map of climatological rainfall.

\section{Spatial variability}

The climatological total divergence at any given location in the tropics will in general be non-zero. Locations characterized by persistent deep convection tend to be convergent in the lower troposphere, while locations characterized by a greater prevalence of shallow convection (considered here to be convection which rarely extends beyond the melting level inversion) tend to be divergent. In the lower troposphere, it has been argued that the total, convective, and background (radiative) divergences are required to go to zero when averaged over spatial scales comparable with the Rossby radius. Here, extensive averaging of many radiosonde profiles $(\sim 2000)$ at individual locations has been used as a surrogate for estimating $\overline{\delta_{b}(p)}$. However, it is not clear why a spatially averaged constraint on the total and convective divergences should give rise to what appears to be a local constraint on the climatological radiative mass flux. One explanation is that the temperature profile at a given location, unlike the divergence itself, is a nonlocal reflection of the influence of all gravity waves within a distance of one Rossby deformation radius.

It is likely that the constraint that $\overline{\delta_{b}(p)}$ be weak reduces to a local constraint on the radiative mass flux only at locations surrounded by homogeneous active deep oceanic convection on a spatial scale larger than one Rossby radius. Figure 8 shows the climatological rainfall pattern in the region of the SPARC Western Equatorial Pacific radiosonde stations. This pattern was obtained by averaging over the $2.5^{\circ}$ by $2.5^{\circ}$ monthly mean rainfall estimates from the Global Precipitation Climatology Project (GPCP) Version 2 Combined Precipitation Data Set (Huffman et al., 1997) from 1991 to 2000. In addition to showing the locations of the Koror, Yap, and Truk radiosonde stations, Fig. 8 also shows the locations of radiosonde stations at Ponape and Majuro. The annual mean static stability and clear sky radiative mass flux profiles of these two stations are shown in Fig. 9. While the climatological rainfall rate at these two locations is quite high, rainfall 


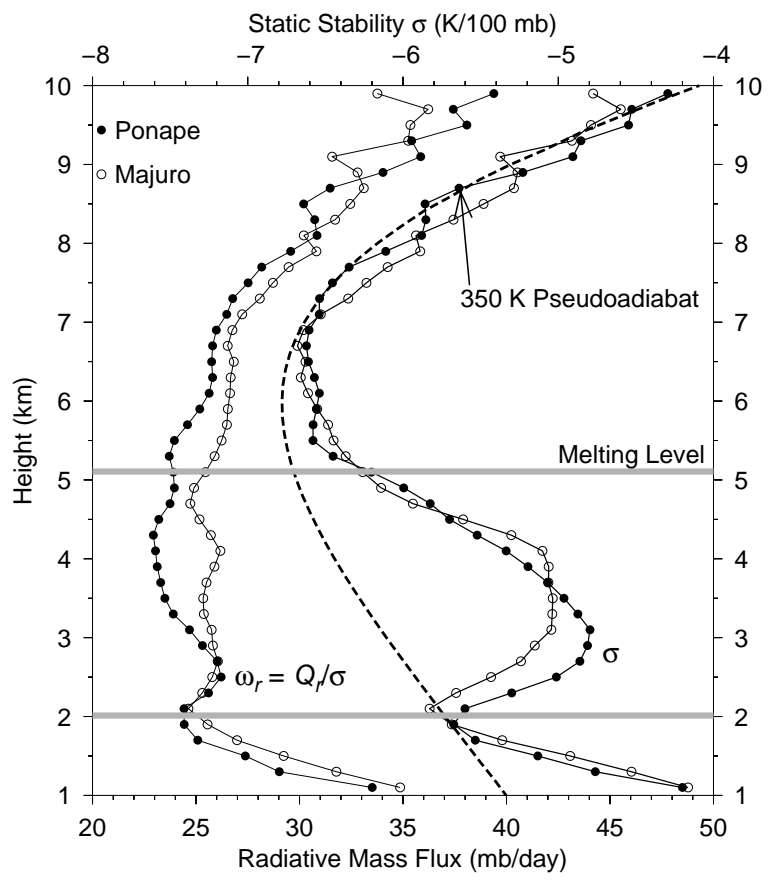

Fig. 9. Annual mean static stability $(\sigma)$ and clear sky radiative mass flux $\left(\omega_{r}\right)$ profiles at Ponape and Majuro. The dashed line is the stability profile of a $350 \mathrm{~K}$ moist pseudoadiabat.

is somewhat more strongly spatially localized at Ponape and Majuro than at the other three radiosonde locations. The constraint on the radiative mass flux divergence may therefore be more weakly enforced at Ponape and Majuro than the other three radiosonde stations, with the result (Fig. 9) that the corresponding radiative mass flux is somewhat more variable with height between $2 \mathrm{~km}$ and $5.2 \mathrm{~km}$.

\section{Uncertainty in the total divergence}

The explanation given here for the weakness of the radiative mass flux divergence in the background atmosphere requires that the total divergence in the lower troposphere effectively go to zero over a spatial scale that is smaller than that of the deep convective region under consideration. However, in the Western tropical Pacific, the climatological rainfall patterns generated by the NCEP, NASA, and ECMWF reanalyses differ significantly from the observed rainfall pattern, and their upper tropospheric divergence patterns differ significantly from each other (Newman et al., 2000). It is likely that the variability of tropical convection in most forecast models is weakly constrained by observations, and highly dependent on the model physics, and especially on their convective schemes and treatment of gravity waves. In principle, it would be desirable to determine the spatial and temporal scale over which the area averaged lower tropospheric total divergence goes to zero from observations. This would re-

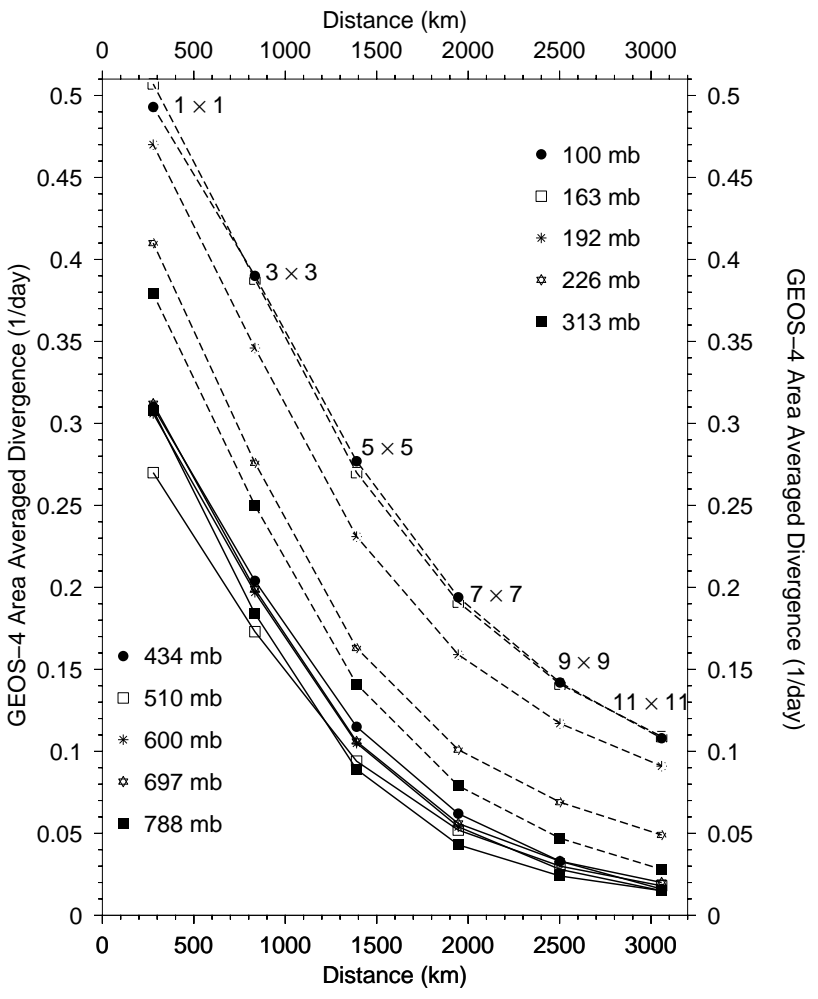

Fig. 10. The rate at which the total divergence, calculated from GEOS-4 assimilated meteorological data, decreases as the spatial scale over which it is being averaged is increased. The GEOS-4 data has a resolution of $2.5^{\circ}$ in the zonal direction and $2.0^{\circ}$ in the meridional direction. The data were taken from July 1, 2001. The divergence was spatially averaged using the approach outlined in the caption to Fig. 3.

quire a sufficiently dense array of rawinsonde data, centered on a deep convective oceanic region of sufficiently large horizontal extent that it exceeded the local Rossby radius. In the absence of such a dataset, it is useful to compare estimates of area averaged total divergence from a number of models. Figure 10 shows the sensitivity of the total divergence to the spatial scale over which it is being averaged using assimilated meteorological data from the Goddard Earth Observing System (GEOS-4) of the NASA Data Assimilation Office. As in Figure 3, divergences in the lower troposphere are weaker and more localized than in the upper troposphere, though the contrast between the upper and lower troposphere is not as strong as in the ECMWF ERA-40 analyses.

\section{Conclusions}

One usually thinks of convective adjustment as a process by which convective heat sources impose a density profile on the background atmosphere. This viewpoint is incomplete, however, because the horizontal and vertical motions forced on the background atmosphere by buoyancy 
anomalies necessarily involve a net entrainment of mass into, or detrainment of mass away, from convective heat sources. Air exchange with the background atmosphere can have a significant impact on the densities within updrafts and downdrafts (especially at warmer temperatures and higher condensate loadings), so there is no reason to anticipate, a priori, that densities within convective plumes should conform to either the undilute reversible adiabatic, or pseudoadiabatic, idealizations.

In the middle troposphere $(5.2 \mathrm{~km}-10 \mathrm{~km})$, lapse rates do follow a moist pseudoadiabatic profile to a high degree of accuracy. Although the reasons for the validity of the pseudoadiabatic approximation in this region of the atmosphere are unclear, it is likely that the rapid decrease in condensate loading of oceanic updrafts just above the melting level (e.g. Jorgensen et al., 1985) is a contributing factor. Not only would this loss of condensate make ascent in these updrafts more consistent with pseudoadiabatic assumptions, it would also tend to reduce the impact of any dry air entrainment (and its associated evaporative cooling) on the temperature and buoyancy of these updrafts.

In the lower troposphere, where lapse rates deviate significantly from both reversible adiabatic and pseudoadiabatic assumptions, the question arises as to whether there exists any simple constraint on tropical temperatures in this region of the atmosphere. It is possible that no such constraint exists, and that the observed mean density profile simply reflect the average condensate loading, and particular rates of entrainment and detrainment, of updrafts and downdrafts in the lower tropical troposphere. In this case, the rates of these small scale processes would not be prescribed by larger-scale considerations. There would be no fundamental explanation for the observed density structure of the lower troposphere, and the cumulus parameterization problem would be more likely to be insoluble.

The paper has motivated a new constraint on temperatures in the lower tropical troposphere by considering the way in which divergence anomalies in the tropics interact with themselves, and with other buoyancy anomalies in their surroundings. The total divergence, averaged over some deep convective region, can be decomposed into a convective divergence, and a divergence associated with radiative cooling of the background atmosphere: $\overline{\delta_{t}(p)}=\overline{\delta_{c}(p)}+\overline{\delta_{b}(p)}$. It was shown, using the ECMWF ERA-40 reanalyses, that the total divergence $\overline{\delta_{t}(p)}$ decays to zero on a spatial scale that, as would be expected, is approximately equal to the Rossby radius of deformation of the gravity waves likely to dominate divergence patterns in the lower tropical troposphere. Ordinarily, one would attribute the finite spatial scale of divergence anomalies in the tropical lower troposphere to two types of damping. One type of damping is associated with an induced divergence in the radiative mass flux of the background atmosphere. It is the usual mechanism whereby buoyancy anomalies influence temperatures in the background atmosphere. Divergence anomalies can also be damped by inducing a compensating divergence in neighboring updrafts and downdrafts, and by generating an exchange of mass with the background atmosphere which has a strong negative feedback on their own buoyancy. The relative importance of the radiative and convective damping mechanisms depends on the timescales associated with each process. Measurements of temperature, water vapor concentration, and condensate loading inside both updrafts and downdrafts have consistently demonstrated that buoyancy anomalies in the lower tropical troposphere are extremely weak, at least over the oceans. This would imply that buoyancy anomalies in the lower tropical troposphere are strongly damped. Radiative transfer calculations show that the climatological clear sky radiative mass flux divergence in the lower tropical troposphere is near zero (Fig. 5). It is therefore likely that the radiative damping timescale in this region of the atmosphere is too long to explain the weakness of observed buoyancy anomalies, and therefore, that convective damping of buoyancy anomalies is highly efficient in the lower tropical troposphere.

The vertical variation of the density in the lower troposphere is an important unsolved problem in tropical meteorology. No model has yet been able to reproduce the detailed structure of the observed lapse rates shown in Fig. 1. Part of the difficulty is that it may involve coupling processes at very different spatial scales: i.e. gravity wave propagation over distances comparable with the Rossby radius, and the small scale effects of turbulent entrainment and detrainment on the buoyancies of updrafts and downdrafts. Although there has been considerable previous discussion on how the stability anomalies near the melting level might be related to the melting or freezing of water (e.g. Johnson et al., 1999), this has been the first attempt to quantitatively account for the vertical variation of lapse rates in the lower tropical troposphere. Clearly, a definitive explanation would involve a more predictive, dynamical model than the diagnostic approach adopted here, where we have made inferences based on the observed density structure, radiative heating rates, and assimilated meteorological datasets. We have also taken the point of view that the melting level denotes a change between two different convective regimes, rather than viewing the lapse rate features as a localized reflection of a heat source that is concentrated in a narrow height range.

The tropics are characterized by a large scale HadleyWalker circulation in which there is a net upper level convective divergence over deep convective regions, and a net upper level radiative convergence over regions in which deep convection is suppressed. This paper had argued that, in the lower troposphere (below the melting level), the circulation cells and associated divergence patterns associated with shallow convection have a much smaller spatial scale, and are balanced almost exclusively by mass flux convergences arising from downdrafts (i.e., that radiative mass fluxes play at best a minor in mass balancing these circulations). 
Deep convective systems are typically convergent in the lower troposphere. (Between 2.0-5.2 km, this convergent influx presumably supplies a downdraft mass flux which increases toward the surface). In this paper, it has been argued that the convective divergence is required to go to zero when averaged over spatial scales comparable with the Rossby radius. In this case, it would be necessary for the lower tropospheric convergence of deep convective clouds to be balanced, on the regional scale, by a divergence from shallow convection. The resulting coupling between deep and shallow convection may be of relevance to the propagation of some tropical convective disturbances, such as the Madden Julian Oscillation (MJO). As it propagates toward the east, the large scale envelope of deep convection associated with an $\mathrm{MJO}$ event is preceded by a zone of shallow convection, which by moistening the lower troposphere up to the melting level (Kemball-Cook and Weare, 2001; Kikuchi and Takayabu, 2004), presumably preconditions the atmosphere for the subsequent deep convection. It may be possible to interpret the low-level divergence of the shallow convection advancing in front of the MJO, in terms of a constraint on the convective divergence, as a response to the low-level convergence of the deep convection within the MJO.

Acknowledgements. ECMWF ERA-40 data used in this study have been provided by ECMWF and obtained from the ECMWF data server. This research was supported by the Natural Sciences and Engineering Research Council of Canada (NSERC), and the Canadian Foundation for Climate and Atmospheric Sciences (CFCAS). This paper has benefited from comments by R. Martin and G. Lesins.

\section{Edited by: U. Lohmann}

\section{References}

Bergman, J. W. and Hendon, H. H.: Calculating monthly radiative fluxes and heating rates from monthly cloud distributions, J. Atmos. Sci., 55, 3471-3491, 1998.

Betts, A. K.: Saturation point analysis of moist convective overturning, J. Atmos. Sci., 39, 1484-1505, 1982.

Bolton, D.: The computation of equivalent potential temperature, Mon. Wea. Rev., 108, 1046-1053, 1980.

Bretherton, C. S. and Smolarkiewicz, P. K.: Gravity waves, compensating subsidence and detrainment around cumulus clouds, J. Atmos. Sci., 46, 740-759, 1989.

Doherty, G. M. and Newell, R. E.: Radiative effects of changing atmospheric water vapour, Tellus, 36 B, 149-162, 1984.

Emanuel, K. A. and Bister, M.: Moist convective velocity and buoyancy scales, J. Atmos. Sci., 53, 3276-3285, 1996.

Folkins, I., and Braun, C.: Tropical rainfall and boundary layer moist entropy, J. Clim., 16, 1807-1820, 2003.
Folkins, I. and Martin, R. V.: The vertical structure of tropical convection, and its impact on the budgets of water vapor and ozone, J. Atmos. Sci., 62, 1560-1573, 2005.

$\mathrm{Fu}$, Q. and Liou, K. N.: On the correlated k-distribution method for radiative transfer in nonhomogeneous atmospheres, J. Atmos. Sci., 49, 2139-2156, 1992.

Gettelman, A., Forster, P. M., Fujiwara, M., et al.: Radiation balance of the tropical tropopause layer, J. Geophys. Res., 109, D07103, doi:10.1029/2003JD004190, 2004.

Giuseppe, F. Di and Tompkins, A. M.: Three-dimensional radiative transfer in tropical deep convective clouds, J. Geophys. Res., 108, D23, doi:10.1029/2003JD003392, 2003.

Holton, J. R.: An Introduction to Dynamic Meteorology, Academic, New York, 1992.

Huffman, G. J., Adler, R. F., Arkin, P. A., et al.: The Global Precipitation Climatology Project (GPCP) Combined Precipitation Data Set, Bull. Amer. Meteor. Soc., 78, 5-20, 1997.

Johnson, R. H., Rickenbach, T. M., Rutledge, S. A., et al.: Trimodal characteristics of tropical convection, J. Atmos. Sci., 12, 23972418, 1999.

Jorgensen, D. P., Zipser, E. J., and LeMone, M. A.: Vertical motions in hurricanes, J. Atmos. Sci., 42, 839-856, 1985.

Jorgensen, D. P. and LeMone, M. A.: Vertical velocity characteristics of oceanic convection, J. Atmos. Sci., 46, 621-640, 1989.

Kemball-Cook, S. R. and Weare, B. C.: The onset of convection in the Madden-Julian Oscillation, J. Atmos. Sci., 14, 780-793, 2001.

Kikuchi, K. and Takayabu, Y. N.: The development of organized convection associated with the MJO during TOGA COARE IOP: Trimodal characteristics, Geophys. Res. Lett., 31, L10101, doi:10.10292004GL019601, 2004.

Lucas, C., Zipser, E. J., and LeMone, M. A.: Vertical velocity in oceanic convection off tropical Australia, J. Atmos. Sci., 51, 3183-3193, 1994.

Mapes, B. E. and Houze, R. A.: Diabatic divergence profiles in Western Pacific mesoscale convective systems, J. Atmos. Sci., 52, 1807-1828, 1995.

Mapes, B. E. and Zuidema, P.: Radiative-dynamical consequences of dry tongues in the tropical troposphere, J. Atmos. Sci., 53, 620-638, 1996.

Mapes, B. E.: Water's two scale heights: The moist adiabat and the radiative troposphere, Q. J. R. Meteor. Soc., 127, 2353-2366, 2001.

May, P. T. and Rajopadhyaya, D. K.: Vertical velocity characteristics of deep convection over Darwin, Australia, Mon. Wea. Rev. 127, 1056-1071, 1999.

Newman, M., Sardeshmukh, P. D., and Bergman, J. W.: An assessment of the NCEP, NASA, and ECMWF reanalyses over the Tropical West Pacific warm pool, Bull. Amer. Meteor. Soc., 81, 41-48, 2000

Rodgers, C. D. and Walshaw, C. D.: The computation of infrared cooling rate in planetary atmospheres, Quart. J. Roy. Meteor Soc., 92, 67-92, 1966.

Wei, D., Blyth, A. M., and Raymond, D. J.: Buoyancy of convective clouds in TOGA COARE, J. Atmos. Sci., 55, 3381-3391, 1998.

$\mathrm{Xu}, \mathrm{K}$. and Emanuel, K. A.: Is the tropical atmosphere conditionally unstable?, J. Atmos. Sci., 117, 1471-1479, 1989. 